msh-mss Mathématiques et sciences humaines

149 | Printemps 2000

Varia

\title{
Charlotte Carcassonne (1938-1999)
}

Charlotte Carcassonne (1938-1999)

\section{Marc Barbut}

\section{OpenEdition}

\section{Journals}

Édition électronique

URL : http://journals.openedition.org/msh/2835

DOI : $10.4000 /$ msh.2835

ISSN : 1950-6821

\section{Éditeur}

Centre d'analyse et de mathématique sociales de l'EHESS

\section{Édition imprimée}

Date de publication : 1 mars 2000

ISSN : 0987-6936

\section{Référence électronique}

Marc Barbut, "Charlotte Carcassonne (1938-1999) », Mathématiques et sciences humaines [En ligne] 149 | Printemps 2000, mis en ligne le 10 février 2006, consulté le 23 juillet 2020. URL : http:// journals.openedition.org/msh/2835; DOI : https://doi.org/10.4000/msh.2835 


\section{CHARLOTTE CARCASSONNE}

$(1938-1999)$

Née Boccon-Gibod, elle est décédée en novembre dernier. Pendant trente-cinq années, elle a joué un rôle majeur dans Mathématiques, Informatique et Sciences humaines.

Après qu'elle eut obtenu le diplôme de statisticien de l'École d'Application de l'INSEE (actuellement École nationale de la statistique et de l'administration économique (ENSAE)), puis celui de l'Institut de statistique de l'université de Paris (ISUP), c'est en 1964 que, sur le conseil de Georges Morlat, elle entra au Centre de mathématiques sociales (CMS) à la $6^{\mathrm{e}}$ section de l'École pratique des hautes études (EPHE), devenue depuis l'École des hautes études en sciences sociales (EHESS).

Il fut d'emblée convenu qu'outre son métier de statisticienne, elle exercerait au CMS la fonction de secrétaire de rédaction de la Revue, créée deux ans plus tôt.

Elle eut ainsi l'essentiel de la responsabilité administrative de Mathématiques, Informatique et Sciences humaines (MISH), et celle des rapports, souvent délicats, avec les auteurs d'articles soumis, et les «referees» sur ces textes. Tâche parfois ingrate qu'elle accomplit toujours avec une bonne humeur, une gentillesse et un doigté qui permit de résoudre au mieux ces problèmes difficiles que pose parfois la gestion éditoriale d'un périodique.

Gestion éditoriale à laquelle elle participa d'ailleurs pleinement en raison de ses compétences scientifiques. Son rôle dépassa largement celui d'une secrétaire de rédaction stricto-sensu.

Bien que gravement malade ces dernières années, elle eut le courage d'assumer ces responsabilités presque jusqu'à la fin.

Pour tout cela, MISH a une immense dette à son égard et gardera toujours en mémoire ce qu'elle fut dans et pour la Revue.

Charlotte Carcassonne fut aussi une enseignante et un chercheur.

L'enseignement la passionnait. Dans les «années soixante», elle fut, pour les mathématiques et la statistique, de l'équipe qui assurait, à titre expérimental et au sein de la $6^{\mathrm{e}}$ section de l'EPHE, l'Enseignement préparatoire à la recherche approfondie en sciences sociales (EPRASS), préfiguration, en beaucoup plus exigeant, des futurs DEA en sciences humaines et sociales. 
Dans les décennies suivantes, c'est dans des Universités, notamment à ParisSorbonne (Paris IV), qu'elle donna, avec un succès à la mesure de l'intérêt qu'elle y prenait, des cours de statistique et d'informatique à des étudiants en sciences humaines.

Elle eut enfin, surtout une féconde activité de recherche, avec une spécialisation!: les applications à la Numismatique de la Statistique!; essentiellement, de la «Statistique circulaire», c'est-à-dire celle dans laquelle la principale des variables analysées est un angle.

À l'origine de cette vocation, une rencontre!: celle de l'antiquisant Julien Guey, spécialiste des monnaies anciennes. En 1968 ou 1969, il vint «à tout hasard» au CMS. pour voir si un statisticien pouvait l'aider à résoudre des problèmes que lui posait l'étude d'un lot de pièces antiques. Ce fut une statisticienne.

De ce premier travail en commun sortit un premier article (réf. [2] ci-dessous). Et ce fut le début d'une longue collaboration, qui allait durer jusqu'à la mort de J. Guey, il y a une dizaine d'années.

Charlotte Carcassonne acquit rapidement une réputation internationale comme statisticienne de la Numismatique. Quelque trente publications en témoignent, qu'elle signait seule (pour les articles sur les méthodes) ou en collaboration avec un archéologue (le plus souvent J. Guey) quand il s'agissait d'exposer une application.

Ces écrits se trouvent pour l'essentiel dans les actes des colloques internationaux de numismatique auxquels elle était invitée (Rome, Barcelone, Louvain-la-Neuve), et dans les revues spécialisées!: Bulletin de la Société Française de Numismatique, Revue Numismatique, Revue Belge de Numismatique - et même, pour trois d'entre elles, dans les publications du Centre d'analyse et de mathématique sociales (notamment dans $\left.M I S H, \mathrm{n}^{\circ} 48,1974\right) ! !$

Points marquant de ce parcours scientifique et pédagogique!:

La thèse de doctorat soutenue en 1984 devant l'Université de Paris-Sorbonne.

La publication subséquente de son livre Méthodes statistiques en Numismatique, complété par l'élaboration d'un logiciel de mise en œuvre de ces méthodes, NUMISMAT.

Charlotte Carcassonne a été l'amie de tous ceux qui ont travaillé avec elle, que ce soit au Centre de mathématiques sociales, dans l'équipe rédactionnelle de Mathématiques, Informatique et Sciences humaines, ou dans d'autres institutions.

Aucun d'entre eux ne l'oubliera. 


\section{PUBLICATIONS DE CHARLOTTE CARCASSONNE}

Livre.

Méthodes statistiques en numismatique, Séminaire de Numismatique Marcel Hoc, Collège Erasme, Louvain-la-Neuve, 1987, 174 p, tables, bibliographie.

\section{Logiciel}

NUMISMAT, programme de statistique pour la numismatique, Paris, EHESS, 1991.

\section{Articles}

[1] Co-rédactrice (sous le nom de C. Chevassus) de «Cahiers Mathématiques-1», ouvrage collectif du CMS, Paris, Gauthier-Villars et Mouton, 1966.

[2] (Avec J. Guey), «Coins de droits et de revers. Études descriptives d'un échantillon», Revue Numismatique, 1970.

[3] «Analyse de variance d'un échantillon d'Antiniani», Bull. Soc. Fr. Numismatique, novembre 1972.

[4] «Monnayage ancien», Revue Numismatique, 1972.

[5] «Angles de droits et de revers, distribution circulaire», Math. Sci. hum., n 48, 1974.

[6] «Le poids de la livre romaine d'après le système de l'As 'libral'», Bull. Soc. Fr. Numismatique, $\mathrm{n}^{\circ}$ 7, 1974.

[7] (Avec M. Christol), «L'aloi de l'Antoninianus dans l'atelier de Cologne sous le règne de Valérien et Gallien - 256-260!: étude statistique», Bull. Soc. Fr. Numismatique, $\mathrm{n}^{\circ}$ 7, 1974.

[8] «Quelques définitions de statistique descriptive», Bull. Soc. Fr. Numismatique, $\mathrm{n}^{\circ} ! 7,1974$.

[9] (Avec F. Dumas), «Comparer deux échantillons!: test de Wilcoxon, test de Student», Bull. Soc. Fr. Numismatique, n 7, 1974.

[10] (Avec F. Dumas et H. Huvelin), «Recherche du poids légal à partir des poids réels. I!: Monnaies médiévales!; II!: Solidi du Bas-Empire», Bull. Soc. Fr. Numismatique, $\mathrm{n}^{\circ}$ 7, 1974.

[11] «Bons et mauvais deniers. Étude statistique de deux échantillons», Numismatique antique, Problèmes et méthodes, Louvain, Peeters, 1975.

[12] «Asymétrie de frai!: Solidi scandinaves», Bull. Soc. Fr. Numismatique, $\mathrm{n}^{\circ} 10$, 1976.

[13] «La livre romaine», Bull. Soc. Fr. Numismatique, n 7, 1977.

[14] (Avec G. Aubin), «Morceaux d'un même trésor!?», Bull. Soc. Fr. Numismatique, $\mathrm{n}^{\circ} ! 7,1977$.

[15] (Avec J. Guey), «Propos de statistique!: quelques échantillons monétaires», Les dévaluations à Rome, Rome, 13-15 novembre 1975, École française de Rome, 1978. 
[16] (Avec J. Guey), «Valeur statistique des petits échantillons», Revue Belge de Numismatique, 1978.

[17] (Avec M. Amandry), «La trouvaille de Folles de Macédoine reconsidérée, test du $\square^{2} »$, Gaz. Numis. Suisse, 29, cahier 116, 1979.

[18] (Avec J. Guey), «Livre romaine, kilo et monnaies», Mélanges de littérature et d'épigraphie latines d'histoire ancienne et d'archéologie, hommage à la mémoire de Pierre Wuilleumier, Paris, Les Belles Lettres, 1980.

[19] «Quelques méthodes statistiques utilisées en numismatique», Revue Belge de Numismatique, 1980.

[20] «Tables pour l'estimation du nombre de coins ayant servi à frapper une monnaie. Méthode du maximum de vraisemblance», Acta del II Symposium Nimismatico de Barcolona, mars 1980, Barcelona, Associacion numismatica Espanola, 1981.

[21] «Quelques méthodes statistiques en numismatique», Statistique et Numismatique, C. Carcassonne et T. Hackens, éds., P.A.C.T.-5, Strasbourg, Conseil de l'Europe, 1983.

[22] «Signification historique. La fiabilité du trésor de 1970», et «Signification numismatique. I!: Caractères externes des monnaies, diamètres, axes. II!: Identités de coins et calcul du volume des émissions», Le Trésor de la Garonne. Essai sur la circulation monétaire en Aquitaine à la fin du règne d'Antonin Le Pieux, R. Etienne et M. Rachet éds., Bordeaux, Fédération historique du Sud-Ouest, 1984, 460 p. [Le prix Allier de Hauteroche a été décerné à cet ouvrage par l'Institut].

[23] (Avec J. Guey), «Les orichalques d'Hadrien dans le Trésor de la Garonne», Mélanges offerts au Professeur M. Labrousse, Toulouse, Presses Universitaires, 1985.

[24] (Avec J. Guey), «Les orichalques d'Hadrien dans le Trésor de la Garonne, 128138 ap. J.-C.», Pallas, Toulouse, 1986.

[25] (Avec J. Guey), «Le Trésor de Lectoure I et le poids de l'antoninianus ou le truqueur vainement traqué», Mélanges offerts au Docteur J.B. Colbert de Beaulieu, Paris, Le Léopard d'Or, 1987.

[26] «Les poids des monnaies des Trésors de Monluzun, Toulouse et Saint-Salvi de Carcavès», Trésors et émissions monétaires du Languedoc et de Gascogne, XII et XIII siècles, Toulouse, Association pour la promotion de l'archéologie et des musées archéologiques en Midi-Pyrénées, 1987.

[27] «Sesterces de Trajan et d'Hadrien dans la circulation», Actes du Colloque Rythmes de la production monétaire de l'Antiquité à nos jours, 10-12 janvier 1986, Louvain, Séminaire de Numismatique Marcel Hoc, 1987.

[28] (Avec A. Davesne), «Le trésor de Gülnar, considérations sur le rythme de la production monétaire au $\mathrm{III}^{\mathrm{e}}$ siècle avant J.-C.», Rythmes de la production monétaire de l'Antiquité à nos jours, 10-12 janvier 1986, Louvain, Séminaire de Numismatique Marcel Hoc, 1987.

[29] (Avec J. Guey), «À propos des tétradrachmes à la Gorgone (C. 545, C. 510 A.C.)», Revue Numismatique, t.XXX, 1988, p. 257-267.

[30] «Étalons, poids légal, pyx, boëtes, remèdes», L’à peu près, Paris, Édition de l'École des hautes études en sciences sociales, 1988. 